\title{
Effects of water stress on photosynthesis, yield, and water use efficiency in winter wheat
}

\author{
Wenhui Zhao ${ }^{1}$, Leizhen Liu ${ }^{1}$, Qiu Shen ${ }^{1}$, Jianhua Yang ${ }^{1}$, Xinyi Han ${ }^{1}$, Feng $\operatorname{Tian}^{1}$, and \\ Jianjun $\mathrm{Wu}^{1}$
}

${ }^{1}$ Beijing Normal University

June 18, 2020

\begin{abstract}
Drought has become one of the major constraints on agricultural development, particularly in areas lacking water. Studying the effects of different water stresses on photosynthesis, growth, yield, water use efficiency (WUE) and water productivity (IWP) of winter wheat will provide scientific irrigation strategies for developing water-saving agriculture. According to the water field capacity, four different water stress levels were set, i.e., 30-40\% of water field capacity (severe stress), 40-50\% (moderate stress), 50-60\% (mild stress) and 60-80\% (well-watered), through an automatic irrigation system by controlling the irrigation amount. The results showed that the diurnal and seasonal changes in photosynthetic parameters such as net photosynthetic rate (Pn), intercellular carbon concentration (Ci), stomatal conductance (Gs), and transpiration (E) significantly decreased under moderate and severe stress. The Pn of mild stress only slightly decreased compared to that of well-watered and was even higher after May 16th. As a result, the dry biomass and 1000-grain weight under mild stress increased $2.07 \%$ and $1.95 \%$ compared with well-watered. Under all water stresses, the heights and straw weights of the winter wheat significantly decreased. It was also found that mild water stress increased the WUE and IWP, which further resulted in the negligible decrease of the fresh weight of the aboveground biomass, dry biomass weight, spike weight, grain weight. Conversely, WUE and IWP significantly decreased under moderate and severe stress, which can affect the growth of winter wheat. So the fresh weight of the aboveground biomass, dry biomass weight, spike weight, grain weight also significantly decreased under moderate and severe stress. Thus, mild stress results in the optimal use of water resources without a significant reduction in yield in the North China Plain. Therefore, mild stress can be considered as a suitable environment for winter wheat growth in arid areas.
\end{abstract}

\section{Hosted file}

Manuscript.doc available at https://authorea.com/users/334608/articles/460571-effects-ofwater-stress-on-photosynthesis-yield-and-water-use-efficiency-in-winter-wheat 